\title{
TRANSFORMATION GROUPS IN THE THEORY OF TOPOLOGICAL LOOPS
}

\author{
SIGMUND N. HUDSON
}

In a recent paper [5] the author has applied the techniques of topological transformation group theory to the study of certain topological loops. The purpose of this note is to show more explicitly the close connection between topological loops and topological transformation groups. It is shown that for every transformation group $G$ which acts reasonably on a space $X$ so that (a) there is a global cross-section $\delta$ from the coset space $G / G_{p}$ into $G$ for $p \in X$, (b) $\delta\left(G / G_{p}\right)$ is a strongly transitive collection of homeomorphisms of $X$, and (c) $G_{p}$ is compact, such a space $X$ may be given a binary operation so that it becomes a topological loop with a left invariant uniformity. Conversely, it is shown that in certain cases a topological loop $L$ allows a transformation group $G$ to act reasonably on $L$ with a cross-section from $G / G_{1}$ for the identity 1 of $L$ satisfying (a) and (b) above. In certain cases (c) will also be satisfied.

The reader may consult $K$. H. Hofmann [3] for the appropriate terminology in topological loops, with the following exceptions. A topological loop $L$ has a left invariant uniformity (see [5]) when there is a uniform structure $\mathfrak{U}$ on $L$ compatible with its topology so that $\mathfrak{U}$ has a base $B$ of entourages satisfying $(x, y) \in B$ if and only if $(a x, a y)$ $\in B$ for all $B \in B$ and $a \in L$. An invariant uniformity is a left invariant uniformity which also satisfies a right invariant condition. If $a$ and $b$ are elements of a loop, the unique solutions $x$ and $y$ to the equations $a x=b$ and $y a=b$ will be designated in this note by $a^{(-1)} b$ and $b a^{(-1)}$, respectively. The parentheses embracing -1 are used as a reminder that $a^{(-1)} b$ is not in general the product of " $a^{-1}$ " and $b$ as in a group. (This is at least the third notation appearing in print for these solutions. At the present this seems to be the most satisfactory.)

The reference for transformation group topics is Montgomery and Zippin [7]. If $G$ is a transformation group acting on a space $X$, and if $G_{p}$ is the isotropy or stability group at $p \in X$, then there is a canonical mapping $\pi: G / G_{p} \rightarrow X$ defined by $\pi\left(g G_{p}\right)=g(p)$. Whenever $\pi$ is a homeomorphism, $G$ is said to act reasonably on $X$ (see [8]). The canonical mapping $\pi$ is continuous, onto, and one-one when $G$ acts effectively and transitively [7, p. 43], but not in general open. A cross-section from $G / G_{p}$ into $G$ is a continuous mapping $\delta$ of the coset

Received by the editors July $30,1963$. 
space into $G$, with $\delta\left(G_{p}\right)$ equal to the identity $e$ of $G$, such that $\phi \delta: G / G_{p} \rightarrow G / G_{p}$ is the identity mapping for $\phi$ the natural projection of $G$ onto $G / G_{p}$. If $G$ acts reasonably on $X$, it is easily seen that $\delta\left(G / G_{p}\right)$ is a transitive collection of homeomorphisms of $X$, and furthermore for $g$ and $h$ in $\delta\left(G / G_{p}\right), g \neq h$ if and only if $g(p) \neq h(p)$. If the stronger condition holds that $g \neq h$ if and only if $g(x) \neq h(x)$ for all $x \in X$ (and all $g, h \in \delta\left(G / G_{p}\right)$ ), then $\delta\left(G / G_{p}\right)$ is said to be strongly transitive on $X$. Strong transitivity is equivalent to saying that $\delta\left(G / G_{p}\right)$ is uniquely transitive on $X$, that is, for $x$ and $y$ in $X$ there is one and only one $g$ in $\delta\left(G / G_{p}\right)$ such that $g(x)=y$.

THEOREM 1. Let $G$ be a topological transformtion group acting reasonably on a space $X$ so that (a) there exists a (full) cross-section $\delta: G / G_{p} \rightarrow G$, (b) $\delta\left(G / G_{p}\right)$ is a strongly transitive collection of homeomorphisms of $X$, and (c) $G_{p}$ is compact. Then $X$ can be given a binary operation so that it becomes a topological loop with a left invariant uniformity.

Proof. Let $f$ denote the evaluation mapping from $G \times X$ into $X$, and let $\pi$ denote the canonical mapping from $G / G_{p}$ onto $X$. Recall that $f$ is continuous and $\pi$ is a homeomorphism. The natural mapping $\phi$ from $G$ onto $G / G_{p}$ is continuous and open.

Define the binary operation on $X$ by the following composition of functions:

$$
X \times X \stackrel{\pi^{-1} \times \mathrm{id}}{\longrightarrow} G / G_{p} \times X \stackrel{\delta \times \mathrm{id}}{\longrightarrow} G \times X \stackrel{f}{\longrightarrow} X,
$$

where id is the identity mapping of $x$; that is, for given $x$ and $y$ in $X$, there exists a unique $g \in \delta\left(G / G_{p}\right)$ such that $\pi(\phi(g))=g(p)=x$, so that the product $x y$ is by definition $g(y)$.

It is easily seen that $p$ is the identity of $X$, because $p x=\delta\left(G_{p}\right)(x)$ $=e(x)=x$, and $x p=g(p)$, where $g=\delta \pi^{-1}(x)$. But $g(p)=\pi \phi \delta \pi^{-1}(x)=x$.

Also solutions to equations exist and are unique: If $x$ and $y$ are in $X$, we wish to find unique $a$ and $b$ so that $x a=y$ and $b x=y$. There exists $g \in \delta\left(G / G_{p}\right)$ such that $g(x)=y$, and only one such $g$ by the strong transitivity condition. Then $b=\pi \delta^{-1}(g)$ is the unique solution desired. Likewise, because $\delta \pi^{-1}(x)=g^{\prime}$ is a homeomorphism of $X$ onto itself, there exists one and only one $a \in X$ such that $g^{\prime}(a)=y$. Then it follows that $x a=y$.

Thus $X$ is an algebraic loop. We have not yet used the fact that $\delta$ is continuous. However when $\delta$ is continuous, then diagram (1) shows that multiplication is the composition of continuous functions and hence continuous. 
We next show that $(x, y) \rightarrow x^{(-1)} y$ is continuous. We have the following:

$$
X \times X \stackrel{\pi^{-1} \times \mathrm{id}}{\longrightarrow} G / G_{p} \times X \stackrel{\delta \times \text { id }}{\longrightarrow} G \times X \stackrel{I \times \text { id }}{\longrightarrow} G \times X \stackrel{f}{\longrightarrow} X,
$$

where $I: G \rightarrow G$ is defined by $I(g)=g^{-1}$. The composition of these functions is continuous and maps $(x, y)$ onto $g^{-1}(y)$, where $g=\delta \pi^{-1}(x)$. But $g^{-1}(y)=x^{(-1)} y$, because $x g^{-1}(y)=g\left(g^{-1}(y)\right)=y$.

Next it is shown that $(x, y) \rightarrow y x^{(-1)}$ is continuous. It is in this section of the proof that compactness of $G_{p}$ is used. We will first prove the following lemma:

Lemma. Let $S$ be a subset of a topological group $G$, and let $f: S \rightarrow G$ be a one-one, continuous mapping. If $f$ is bounded, i.e., the closure of $\left\{f(x)^{-1} x: x \in S\right\}$ is compact, then $f$ is a homeomorphism from $S$ to $f(S)$.

Proof. It is sufficient to show that, if $\left\{x_{p}\right\}$ is a net in $S$ such that $\lim _{p} f\left(x_{p}\right)=a \in f(S)$, then any subnet $\left\{x_{q}\right\}$ of the net $\left\{x_{p}\right\}$ has a further subnet converging to $f^{-1}(a)$. There is a subnet $\left\{x_{r}\right\}$ of $\left\{x_{q}\right\}$ such that $c=\lim _{r} f\left(x_{r}\right)^{-1} x_{r}$ exists; thus $\lim _{r} x_{r}$ exists and is equal to $a c$ by continuity of multiplication. Furthermore, $f^{-1}(a)=f^{-1}\left(\lim _{r} f\left(x_{r}\right)\right)$ $=f^{-1}\left(f\left(\lim _{r} x_{r}\right)\right)=a c$. Thus $\left\{x_{r}\right\}$ is the desired subnet of $\left\{x_{q}\right\}$ converging to $f^{-1}(a)$. Returning to the proof of the theorem, define the following space and functions: $G \times G$ is the product topological group obtained from $G$ with coordinate-wise multiplication, $\sigma \times \sigma: L \times L$ $\rightarrow G \times G$ is defined by $\sigma \times \sigma(x, y)=(\sigma(x), \pi(y)), \mu: X \times X \rightarrow X \times X$ is defined by $\mu(x, y)=(x y, y), m^{*}: G \times G \rightarrow G \times G$ is defined by $m^{*}(g, h)$ $=(g h, h)$, and $\gamma: X \times X \rightarrow G \times G$ is the composite function $\sigma \times \sigma$ followed by $m^{*}$. It is easily verified that $\sigma \times \sigma$ and $m^{*}$ are homeomorphisms, and $\mu$ is one-one and continuous. The following diagram illustrates the situation:

$$
\begin{aligned}
& X \times X \stackrel{\sigma \times \sigma}{\longrightarrow} G \times G \stackrel{m^{*}}{\longrightarrow} G \times G \\
& \mu \downarrow \\
& X \times X \stackrel{\sigma \times \sigma}{\longrightarrow} G \times G .
\end{aligned}
$$

There is a unique function $z: \gamma(X \times X) \rightarrow G \times G$ so that the diagram is commutative. Routine calculation shows that, for $v \in \gamma(L \times L)=S$, $z(v)^{-1} v$ belongs to $\left(G_{p}, e\right)$, which is a compact subset of $G \times G$. The above lemma implies that $z$ is a homeomorphism, making $\mu$ a homeomorphism. Openness of $\mu$ implies continuity of $(x, y) \rightarrow y x^{(-1)}$.

Finally, compactness of $G_{p}$ implies that the coset space $G / G_{p}$ has a uniformity invariant under the action of $G$ [4], that is, $X$ has a left 
invariant uniformity because the elements of $\delta\left(G / G_{p}\right)$ are the left translates.

It is remarked that the author tried at one time (unsuccessfully) to show that $(x, y) \rightarrow y x^{(-1)}$ was continuous without assuming compactness of $G_{p}$, but assuming local compactness of $X$. With the stronger assumptions of either compactness for $X$ or a Euclidean neighborhood in $X$, it can be shown that this inverse function is continuous.

Partial converses to Theorem 1 are now given.

Theorem 2. Let $M$ be a topological loop. In the following cases there exists a topological transformation group $G$ acting reasonably on $M$ with respect to $G_{1}$ for the identity 1 of $M$, and there is a cross-section $\delta: G / G_{1}$ $\rightarrow G$ so that $\delta\left(G / G_{1}\right)$ is strongly transitive on $M$ :

Case 1. $M$ is locally compact.

Case 2. $M$ has an invariant uniformity.

Proof of Case 1. For each $x \in M$, let $L_{x}$ denote the homeomorphism of $M$ onto itself mapping $y$ onto $x y$. Let $G$ be the group generated by $\left\{L_{x}: x \in M\right\}$ in the group of all homeomorphisms of $M$ onto itself. Let $G$ have the $g$-topology [1]. In [5] $G$ is called the group generated by the left translates of $M$, and it is pointed out that $G$ is a topological group of homeomorphisms with the $g$-topology acting effectively and transitively on $M$. From the proof of Theorem 4 of [5] it may be inferred that the mapping $C_{L}: M \rightarrow G$ defined by $C_{L}(x)=L_{x}$ is a continuous function. Let $\phi: G \rightarrow G / G_{1}$ and $\pi: G / G_{1} \rightarrow M$ be the natural and canonical mappings (as in Theorem 1 above). Then $\pi \phi$ restricted to $C_{L}(M)$ equals $C_{L}^{-1}$, and $\pi^{-1}=\phi C_{L}$. Thus $C_{L}$ is a homeomorphism and $\pi$ is open. It is known that $\pi$ is continuous, one-one, and onto. Hence $G$ acts reasonably. Also $C_{L} \pi$ is a homeomorphism of $G / G_{1}$ into $G$ and is easily seen to be a cross-section. Finally, strong transitivity is equivalent to the condition that $L_{a}(x)$ $=L_{b}(x)$ if and only if $a=b$, a condition always satisfied in a loop.

Proof of CASE 2. Let $G$ again denote the group generated algebraically by the left translates of $M$, but let $G$ have the topology of uniform convergence [6, p. 226]. Because of the invariant uniformity condition, each left translate and its inverse, and consequently each element of $G$ is a uniformly continuous mapping with respect to the invariant uniformity. Hence $G$ is a topological transformation group [2] of homeomorphisms acting effectively and transitively on $M$. Now we will show the mapping $C_{L}$ is continuous. Let $B$ be an entourage in the uniformity for $G$ of the form $B=\{(f, g): f$ and $g \in G$ and $(f(x), g(x)) \in B^{\prime}$ for all $\left.x \in M\right\}$, where $B^{\prime}$ is an entourage which is 
invariant. If $(y, z) \in B^{\prime}$, then $\left(C_{L}(y), C_{L}(z)\right) \in B$, because $(y x, z x)$ $=\left(L_{y}(x), L_{z}(x)\right)$, and because $(y x, z x) \in B^{\prime}$ if and only if $(y, z) \in B^{\prime}$. Since entourages of the type of $B$ form a base for the uniformity on $G, C_{L}$ is uniformly continuous. As in Case 1 , it follows that $C_{L}$ is a homeomorphism, $\pi$ is a homeomorphism, $C_{L} \pi$ is a cross-section of $G / G_{1}$ into $G$, and $G$ acts reasonably. Again $C_{L} \pi\left(G / G_{1}\right)$ is a strongly transitive collection of homeomorphisms of $X$.

In general neither $G$ as defined above ( $G$ with the $g$-topology or $G$ with the topology of uniform convergence) is locally compact; however, it is sometimes convenient to have a locally compact group containing the translates and acting on $M$. In Case 1 above the closure $G^{*}$ of $G$ in the space of all continuous functions from $M$ to $M$ with the $g$-topology is the desired group when it is further assumed that $M$ is connected and has a left invariant uniformity. The situation is more completely described in the next theorem.

Theorem 3. Let $M$ be a locally compact, connected loop. Let $G$ be the group generated by the left translates, let $G^{*}$ be the closure of $G$ in the space of all continuous functions from $M$ to $M$, and let $G_{1}^{*}=\left\{g \in G^{*}: g(1)=1\right\}$, all with the g-topology. Then the following are equivalent:

(a) $G_{1}^{*}$ is compact,

(b) $G_{1}^{*}$ is compact and $G^{*}$ is a transformation group of $M$,

(c) $M$ has a left invariant uniformity,

(d) $G$ is a uniformly equicontinuous collection of homeomorphisms,

(e) $G^{*}$ is a locally compact transformation group of $M$ and $G_{1}^{*}$ is compact.

When, for example, (b) holds, then $G^{*}$ acts reasonably on $M$, there is a natural cross-section from $G^{*} / G_{1}^{*}$ into $G^{*}$, and the strong transitivity condition is satisfied with respect to this cross-section.

Proof. It is known that $G_{1}^{*}$ is a topological semigroup. We first show that $G_{1}$ is dense in $G_{1}^{*}$. It is easily seen that each element $g^{\prime}$ of $G$ can be represented uniquely as $g^{\prime}=L_{x} h$, where $h \in G_{1}$ and $x$ is some element of $M$. Let $g \in G_{1}^{*}$. There exists a net $\left\{g_{t}\right\}$ with each $g_{t}$ in $G$ so that $\left\{g_{t}\right\}$ converges to $g$. Let $L_{x_{t}} h_{t}$ be the representation for $g_{t}$, where $h_{t} \in G_{1}$. Then $\left\{x_{t}\right\}$ converges to $g(1)=1$. Hence $\left\{h_{t}\right\}$ converges to some $h \in G_{1}^{*}$ and $\left\{L_{x_{t}}\right\}$ converges to $L_{1}$. Thus $\left\{L_{x_{t}} h_{t}\right\}$ converges to $L_{1} h$ and $\left\{h_{t}\right\}$ converges to $h=g$, implying that $G_{1}$ is dense in $G_{1}^{*}$.

In the argument just concluded the fact that $G=A G_{1}$, where $A=\left\{L_{x}: x \in M\right\}$, was used. It is also easily shown that $G^{*}=A G_{1}^{*}$.

We will show that each condition in the statement of the theorem implies the following one. Note that (e) trivially implies (a). If $G_{1}^{*}$ 
is compact, then $G_{1}^{*}$ is a compact topological semigroup with the dense subgroup $G_{1}$. It follows that $G_{1}^{*}$ is a topological group [9], and, consequently, that $G^{*}$ is also a topological group in the $g$-topology and (b) is satisfied. It then follows that the coset space $G^{*} / G_{1}^{*}$ has a uniformity invariant under the action of $G^{*}[4]$. But $G^{*} / G_{1}^{*}$ may be identified topologically with $M$ (when condition (b) is satisfied, one may construct, as in Theorem 2, the cross-section from the coset space $G^{*} / G_{1}^{*}$ into $G^{*}$ ). Hence $M$ has a left invariant uniformity. This means that $G$ is a uniformly equicontinuous collection of homeomorphisms with respect to this uniformity (see the proof of Theorem 1 in [5]). However if $G$ is a uniformly equicontinuous collection, then Arens' Theorem in [1] shows that $G^{*}$ is a locally compact transformation group of $M$; and a modification of Dieudonné's Proposition 12 in [2] shows that $G_{1}^{*}$ is compact. This concludes the proof.

We remark that in the situation of Theorem 3 both $G$ and $G^{*}$ are connected, as shown by Theorem 2 of [5].

The author is grateful to $\mathrm{K}$. H. Hofmann for his suggestions, particularly in connection with Theorem 1 and its lemma.

\section{REFERENCES}

1. R. Arens, Topologies for homeomorphism groups, Amer. J. Math. 68 (1946), 593-610.

2. J. Dieudonné, On topological groups of homeomorphisms, Amer. J. Math. 70 (1948), 659-680.

3. K. H. Hofmann, Topologische Loops, Math. Z. 70 (1958), 13-37; Topologische Loops mit schwachen Assoziativitätsforderungen, ibid. 70 (1958), 125-155; Topologische Doppelloops, ibid. 70 (1958), 213-230.

4. S. N. Hudson, $A$ note on invariant uniformities in coset spaces, Math. Scand. 12 (1963), 36-38.

5. - Topological loops with invariant uniformities, Trans. Amer. Math. Soc. 109 (1963), 181-190.

6. J. L. Kelley, General topology, Van Nostrand, Princeton, N. J., 1955.

7. D. Montgomery and L. Zippin, Topological transformation groups, Interscience, New York, 1955.

8. P. S. Mostert, Reasonable topologies for homeomorphism groups, Proc. Amer. Math. Soc. 12 (1961), 598-602.

9. A. D. Wallace, The structure of topological semigroups, Bull. Amer. Math. Soc. 61 (1955), 95-112.

SYRACUSE UNIVERSITY AND

Mathematisches Institut DER Universität, Tübingen, Germany 